\title{
Design and Implementation of Smart Camera Network for Efficient Wide Area Surveillance
}

\author{
Soomi Yang \\ Department of Information Security, The University of Suwon \\ smyang@suwon.ac.kr
}

\begin{abstract}
For efficient wide area surveillance, smart cameras should provide large observed data and many intelligent analysis functions. In order to extend the surveillance area and overcome occlusion, integration of data from multiple cameras and post analysis of it are required. The surveillance data includes low level multimedia data, recognized feature data, event alarms and others. Integration of those data needs interaction between cameras. This paper presents the design of smart camera network and the distributed data management scheme such as data structuring, interchanging and deployment. An overwhelming amount of surveillance data is generated by smart cameras. The data contained in them if properly structured, integrated and analyzed, can induce useful context information. This work builds a hierarchical data deployment structure and exchange related data arriving to and from multiple smart cameras. Proactive data deploying provides the main concepts and properties to model a hierarchical data structure. Our simulation and implementation of the strategy shows efficiency and usefulness.
\end{abstract}

Keywords: Smart camera network, Surveillance data deploying, Reasoning based on ontology, Wide area surveillance System, Integrated context inference

\section{Introduction}

Smart camera network provides geographically distributed large scale sensor data and many intelligent functions for wide area surveillance. It utilizes Internet which is different from private network of CCTV (Closed Circuit Television) network. The latency time of the Internet makes it difficult to access quickly and efficiently to such huge and widely distributed data. For the surveillance of the large area, agents built in smart cameras should collaborate through integration of data. Surveillance data includes audio, video, recognized feature data, environment ontology data, event alarms and others. Because of the latency of Internet involved, and the requirements of real time play out a framework for the working of such a distributed data management system considering administration hierarchy to help law enforcement is needed. Distributed agents within smart cameras receiving heterogeneous data from various sources have autonomy, collaborate with each other, and carry out analysis based on distributed knowledge bases. A framework for the integration of surveillance data supplied by a set of agents is designed. The agents in the same level are interconnected through a peer network. And non-leaf administrative agents in a geographic area hierarchy or in an administration hierarchy build a hierarchical structure according to their law enforcement hierarchy. This framework guarantees the consistency and expressivity of the environment data used in the data integration process. Before the exchange of analysis, each agent may process the consolidated data for the integrated analysis. It 
helps security persons by giving appropriate prediction or decision based on the analysis result.

The distribution of demands for surveillance data is often skewed, and the surveillance devices have different capabilities. These can lead to poor data communication and dropped messages. In this paper, we propose a proactive data deploying scheme for efficiency and interoperability. Implementation is also going on into our distributed surveillance network environment.

The rest of the paper is organized as follows. Section 2 briefly surveys related work of distributed camera network. Our proposed modeling of scalable surveillance infrastructure is explained in Section 3. Section 4 describes the adaptive surveillance data management technique for efficient camera network deploy. In Section 5, implementation results are presented. Finally, Section 6 concludes with an outline of our future work.

\section{Related Work}

There has been a lot of multimedia data management schemes proposed with analogical peers. [1,2] dealt with multimedia data which is the most interested data format for the surveillance environment. [1] designed PI(Proportional/Integral) controller to meet QoS(Quality of Service) requirements. [2] utilized multimedia server cache. In our distributed surveillance environment, multimedia data is generated continuously and analysis should be done in real time. In [3, 4], a cooperative caching framework is introduced and claimed to be effective to data availability. In [4], a replica allocation method and clustering in distributed networks are introduced to improve data accessibility in a mobile communication environment. Another cooperative data management scheme for similar peers is described in [5], which combines the $\mathrm{P} 2 \mathrm{P}$ communication technology with a conventional mobile system.

To ease merging of heterogeneous surveillance data, effort for the standardization for physical security is done by ONVIF (Open Network Video Interface Forum) [6] and PSIA(Physical Security Interoperability Alliance) [7]. They define, recommend, and promote standards for IP-based security products. [8] introduces wide area surveillance networks based on ONVIF standard.

In this paper, we consider efficient surveillance infrastructure with novel data management scheme. And our implemented system tries to meet the requirements of industry by providing functions recommended by ONVIF standard organization.

\section{Scalable Surveillance Infrastructure}

Our smart camera network refers to the networking infrastructure that is designed to provide pervasive and reliable access to data and resources over wide area network utilizing Internet, across organizational domains. It connects hundreds of geographically distributed smart cameras, other sensors and storage resources located in different parts of the nation to facilitate sharing of data and resources.

For the creation of wide area surveillance network, we should develop a protocol among local surveillance network. The efficiency of their cooperation depends on the efficiency of the protocol. To offer high data availability, low bandwidth consumption, increased fault tolerance, and improved scalability of the overall system, we introduce a set of data management services and protocols. The architecture consists of a hierarchy of servers, which contains a number of non-leaf node administrative servers and leaf node smart cameras, as shown in Figure 1. This figure shows the different hierarchy of the national network for surveillance. To address scalability, they are organized in a combination of hierarchical and 
flat topologies that represent propagation graphs that minimize inter node communication costs. It has 5 levels. From the top, they are the national servers, regional servers, city servers, organization servers and the smart camera systems respectively. The first 3 levels are responsible for receiving and processing data from the $4^{\text {th }}$ level. In addition, in these levels law enforcements can gain access the recordings and other analyzed data. In the $4^{\text {th }}$ level, organizations may have one or more smart camera systems. It is responsible for transmitting image recordings, recognized feature data, context data and event alarms extracted from smart camera agents to the city servers at the $3^{\text {rd }}$ level.

To address efficiency and scalability, for the hierarchical topology, we have higher network bandwidth between the servers in the same administrative institution. We use the mesh topology with the higher network bandwidth and they form cluster.

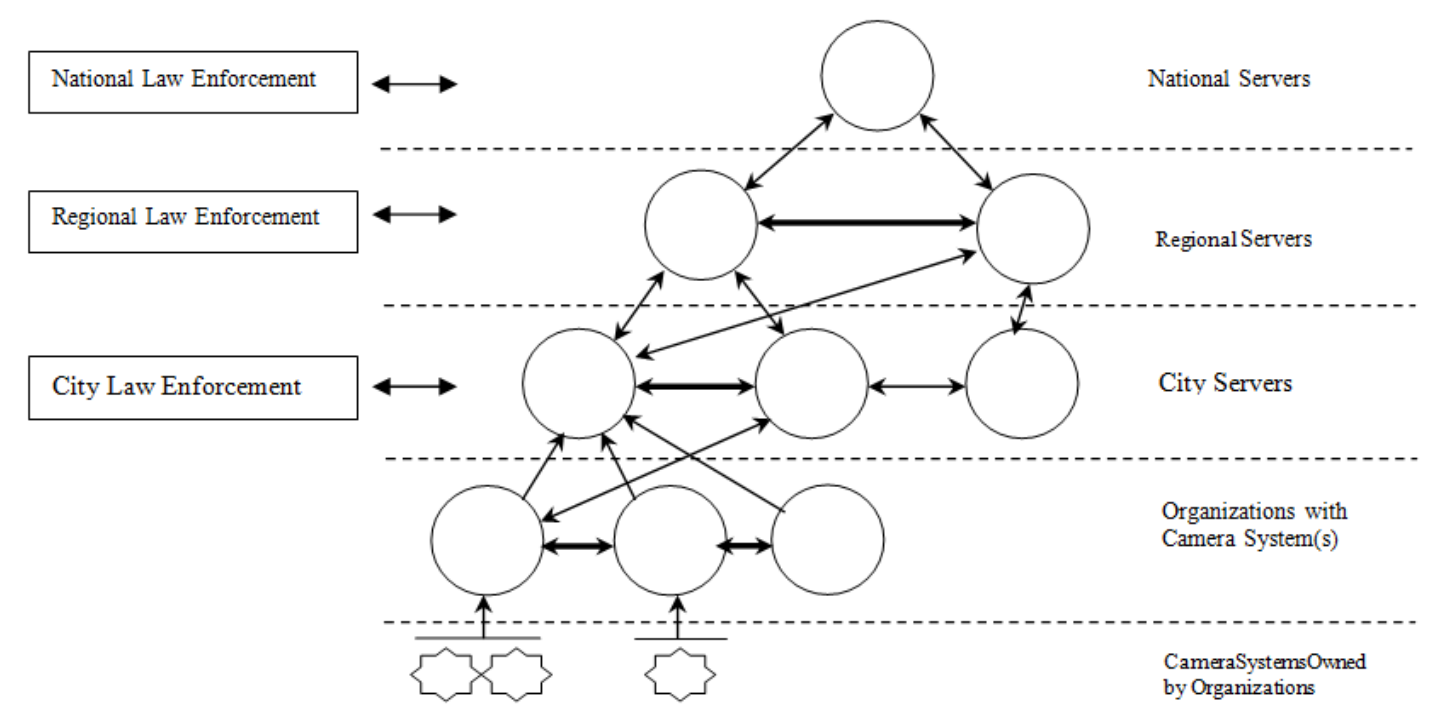

Figure 1. Surveillance network architecture

The servers form a graph structure. They can communicate each other freely within access control permission to perform their own local analysis based on their own local knowledge base. For the higher level integrated analysis which includes several cameras of larger area, they use deployed data of others.

Various data, such as video, feature data including biometrics, event alarms, timestamps, GPS (Global Positioning System) location coordinates, and other information originate from many kinds of input devices. These data, often referred to as institutional, regional, and national data depending on the hierarchy of the administrative servers operating them, is integrated with each other so as to analyze and deliver efficiently the requested result to the users.

With a data sharing and integration, even small servers, owing to the help of higher level servers, can achieve very high hit rate locally, thus reducing significantly the bandwidth requirements of the network connection and the latency perceived by the users. To pursue this performance gain, the deploying of surveillance data in administrative servers and proper distribution structure is needed for the better hit rates achievable by keeping surveillance data in local data store within peer cluster network. 


\section{Adaptive Surveillance Data Management}

The administrative agent's data store might be more heavily loaded as the number of data increases faster and their update intervals go shorter and shorter. The situation becomes worse as more data are replaced by multimedia data. This then cause the middle level agents to suffer a long period of time before they contain most of the data needed in the surveillance camera network, degrading the whole system performance significantly.

Therefore a proactive, rather than reactive, deploying of surveillance data is needed. By proactive deploying, it means that the middle level agents cooperate with the master in exchanging necessary information on the data size, the request rate, and so on enabling the master to fetch and broadcast the data before a lower level agents requests it.

As shown in Figure 1, the surveillance data is distributed over the peer servers. For the higher level integrated analysis they need to carry out the indexing and retrieval of the information distributed across the servers in an efficient manner. To aid the task, data deployment decisions are made based on a cost model that evaluates data access costs and performance gains of creating each replica. The estimation of costs and gains is based on factors such as run-time accumulated read/write frequency statistics, response time, network bandwidth, and replica size.

We calculate the weight of data to measure its relative importance as proposed in [10]. The higher the weight, the lower is the probability of the data being replaced. We also use different policy in which the weight is proportional to the network bandwidth. The network bandwidth is different between servers as shown in Figure 1. The weight $w$ is computed as following, where $F$ is the number of times the data is accessed, $S$ is the size of the data compared to network bandwidth and $R$ is the recency proportional to the update interval. The three exponents $f, r$ and $s$ are weighting factor and the absolute values of them should be adjusted in accordance with the relative importance.

$$
\begin{aligned}
& \begin{array}{l}
w=F^{f} S^{s} R^{r} \\
=\left(c \cdot n_{k} \frac{\sigma}{i^{\alpha}}\right)^{f} \cdot\left(B_{k} \cdot \sum_{i \in D} S_{i}\right)^{s} \cdot\left(\frac{L_{j}}{V_{j}} \mathrm{P}\left(\mathrm{d}_{\mathrm{j}} \mid D\right)\right)^{r}
\end{array} \\
& \text { where } \sigma=\left(\sum_{i \in D} 1 / i^{\alpha}\right)^{-1}
\end{aligned}
$$

In Equation (1), $c$ is a coefficient for the number of server $n_{k}$ and $\alpha$ is a constant close to 1 according to the Zipf's law [12] for $\mathrm{D}=\{1,2, \ldots,|\mathrm{D}|\}$ as the whole set of surveillance data including recognized features of the target, and assume that the data in $D$ are ranked in the order of their suspiciousness or importance. $B_{k}$ is the network bandwidth and $S_{i}$ is the size of the $i$-th surveillance data. $L_{j}$ and $V_{j}$ is the travel length and velocity of the target $j$. Using Bayes' theorem, searching a target of interest having $d_{j}$, reduces to finding the maximum of the $P(d j \mid D)$.

$L_{j}$ and $V_{j}$ can be computed from the smart camera's PTZ(Pan Tilt Zoom) values offered by ONVIF functions, timestamp and coordinate values from the map. The range of PTZ values are 0 360, -5 185, 1 37. As shown in Figure 2, when we let $C$ be a location of a camera and $O$ be a location of an object, the distance between the camera and an object can be calculated from the Equation (2).

$$
\overline{O C}=H \cdot \sec \theta_{h}
$$




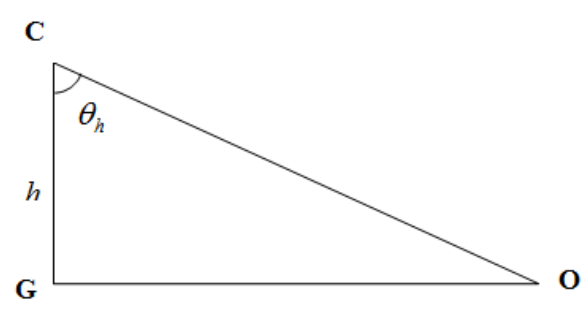

(a)

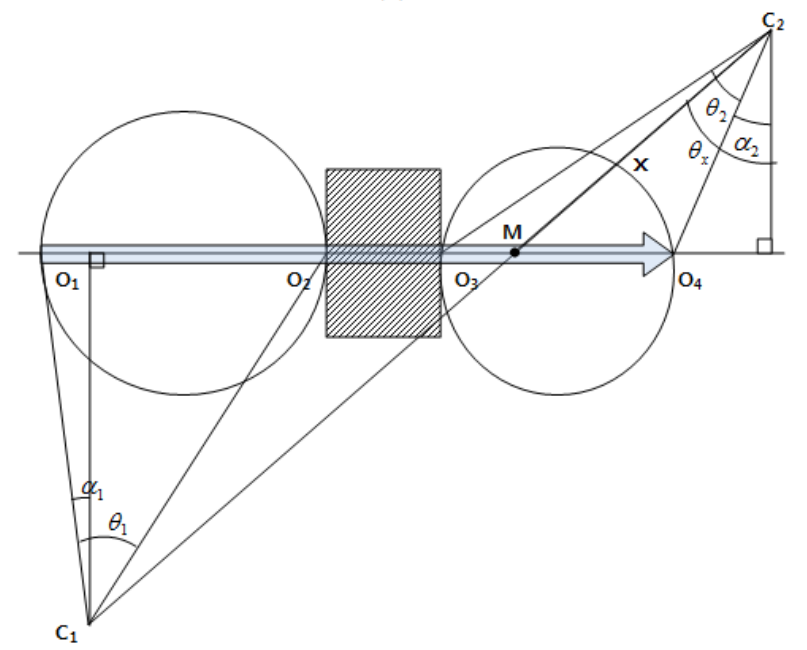

(b)

Figure 2. Models for the calculation of direction and velocity: (a) one camera with height $h$; (b) two cameras with occlusion area

If we let $a=\overline{O_{1} C_{1}}, b=\overline{O_{2} C_{1}}, c=\overline{C_{1} C_{2}}, d=\overline{O_{3} C_{2}}, e=\overline{O_{4} C_{2}}$, the distance object moved between two cameras containing occlusion area can be calculated from Equation (3)

$$
\begin{aligned}
\overline{O_{1} O_{4}} & =\overline{O_{1} O_{2}}+\overline{O_{2} O_{3}}+\overline{O_{3} O_{4}} \\
& =\overline{O_{1} O_{2}}+\overline{O_{2} M}-\overline{O_{3} M}+\overline{O_{3} O_{4}} \\
& =\sqrt{a^{2}+b^{2}-2 a b \cdot \cos \theta_{1}}+\sqrt{b^{2}+(c-x)^{2}-2 b(c-x) \cdot \cos \left(\theta_{x}+\alpha_{1}-\theta_{1}\right)} \\
& -\sqrt{d^{2}+x^{2}-2 d x \cdot \cos \left(\theta_{2}+\alpha_{2}-\theta_{x}\right)}+\sqrt{d^{2}+e^{2}-2 d e \cdot \cos \theta_{2}}
\end{aligned}
$$

where $x=\overline{C_{2} M}, \theta_{x}=\angle M C_{2} H_{2}$

If the probability of having cached data is $q$, the average expected packet transmission can be computed as following Equation (4). 


$$
E(t)=(1-q) \cdot\left(l \cdot \frac{1}{1-p}\right) \cdot\left(w \cdot \frac{1}{h}\right)
$$

where $E(t)$ means the expectation of the packet traffic, $l$ means the latency, $p$ means the probability of packet loss and $h$ is the number of hops.

If we let the probability of data miss according to a Poisson process with the same rate to keep the number of data miss in the system roughly constant, the probability of having data miss at each server, $p$ can be computed. We can reduce the data miss probability $p$, with proactive data deploying according to the previously calculated $w$. Consequently it reduces the packet traffic $E(t)$.

\section{Simulation}

We simulated our model to evaluate the performance. We carry out a simulation to count the number of packet transmissions for accomplishing integrated reasoning. For the rate parameter $\lambda$ and given the packet loss ratio $p$, we can inspect the effects to the average packet transmission. Figure 3 shows the expected packet transmission decrement ratio by $\lambda$ and $p$. When the packet loss is small, the difference is small. However, as the packet loss gets bigger, it suffers more increasing packet transmission without our proposed adaptive data management policy.

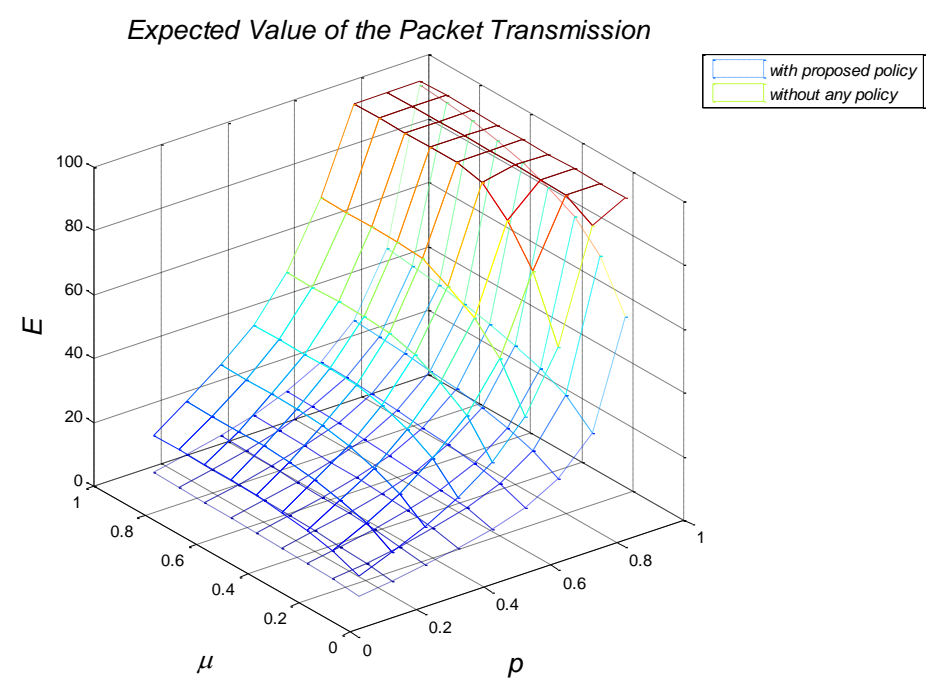

Figure 3. The expected value of the packet transmission

\section{Implementation}

We implemented our cooperative framework for integrating surveillance data with an adaptive deploying technique into our distributed surveillance system. Figure 3 shows a portion of our testbed structure. We provide Android client interface in addition to web interface. Although ONVIF camera supports MPEG-4, H.264, MJPEG, it needs transcoding for Android application. We use VLC media player[11] for video source transcoding. 


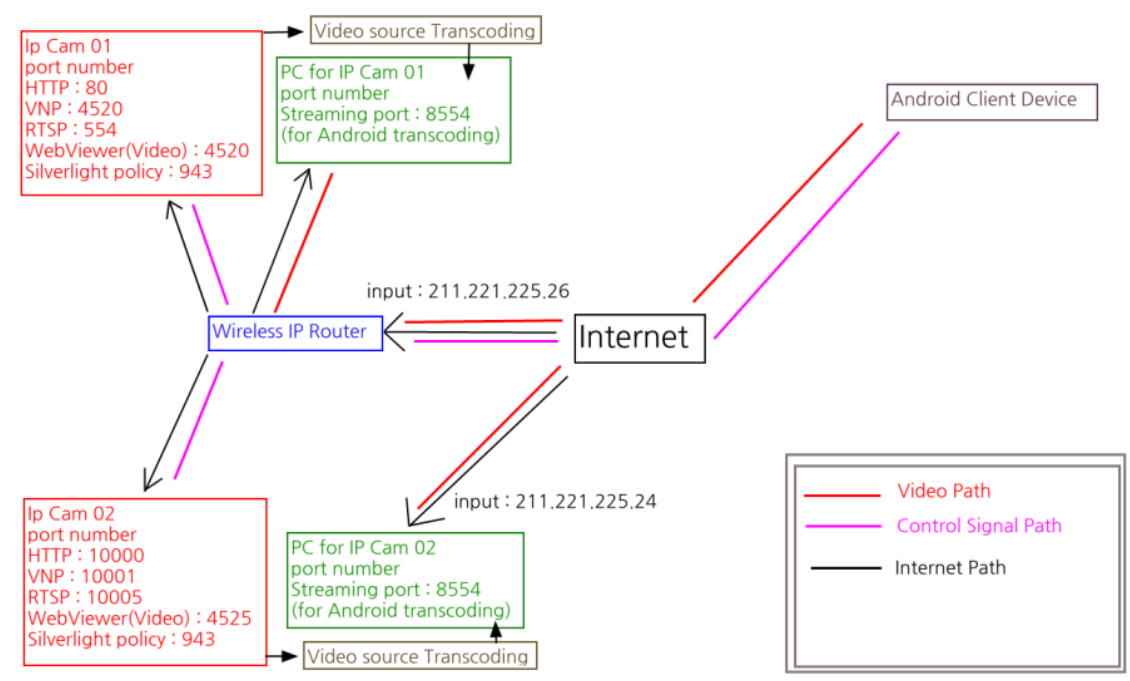

Figure 3. Part of testbed structure

Figure 4 shows parts of mobile user interface which provide map and control panel. Security person can respond promptly and easily with this smart phone interface. They can inquire real time video or stored surveillance data. And they can request smart camera control or other pertinent action through defined queries.
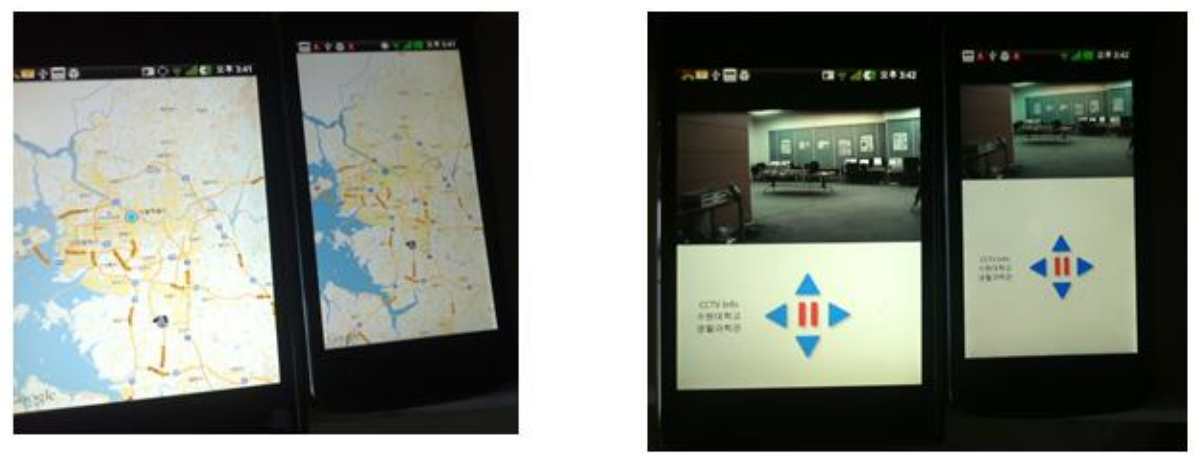

Figure 4. Mobile interface for various Android smart phone

\section{Conclusion}

We describe the design and implementation of smart camera network for wide area surveillance. In such a distributed surveillance environment, surveillance devices such as smart cameras may carry heterogeneous video data, recognized feature data, analysis result data, event alarms and others with different transmission ranges, latency, and formats. These devices not only can get services from a region server, but also they can form and generate a peer cluster to provide services efficiently to each other. For such a camera network, an effective surveillance data deploy framework that can handle heterogeneous devices is required. In this paper, we propose a flexible deployment scheme which is adaptive to the actual data demands. Our scheme uses conformity to update and share data in a cooperative way. The proactive deploying of surveillance data maximizes the agent's potential for making analysis from operating the 
hierarchical structure data distribution. The surveillance data deploying structure, where the data are deployed within the cluster in the order of hierarchy and in the direction of suspect respectively, are formulated and optimal service policy is pursued in view of which middle level agents to be included in the system. To investigate how the optimal deploying scheme, we formulate the weight of each data to improve performance and make service policy respond to system parameter changes. The system parameters of our interest include data size, frequency, data update interval, the network distances between agents, and some relevant factors. Finally, simulation and implementation results are discussed in Section 5. More realistic data will be given in future work. Our scheme shows the efficiency of data deploying resulted in better data integration and analysis.

\section{Acknowledgements}

This work is supported by the GRRC program of Gyeonggi province. [GRRC SUWON2012-B1, Cooperative CCTV Image Based Context-Aware Process Technology].

\section{References}

[1] W. He and K. Nahrstedt, "End-to-End Delay Control of Multimedia Applications over Multihop Wireless Links", ACM Transactions on Multimedia Computing, Communications and Apoplications, vol. 5, no. 2, Article 16, (2008).

[2] T. R. Go. Nair and P. Jayarekha, "A Rank Based Replacement Policy for Multimedia Server Cache Using Zipf-Like Law", Journal of Computing, vol. 2, Issue 3, (2010).

[3] L. Yin and G. Cao, "Supporting Cooperative Caching in Ad Hoc Networks", IEEE Tr. On mobile computing, vol. 5 , no. 1, (2006).

[4] S. Borst, V. Gupta and A. Walid, "Self-organizing algorithms for cache cooperation in content distribution networks", Bell Labs Technical Journal, (2009).

[5] K. F. Yeung and Y. Yang, "Mobile information retrieval in a hybrid peer-to-peer environment", Mobility '09 Proceedings of the 6th International Conference on Mobile Technology, Application \& Systems, (2009).

[6] ONVIF (Open Network Video Interface Forum), http://www.onvif.org.

[7] PSIA (Physical Security Interoperability Alliance Specification Package Q12009, http://www.psiaalliance.org.

[8] T. Senst, M. Patzold, R. H. Evangelio, V. Eiselein, I. Keller and T. Sikora, "On Building Decentralized WideArea Surveillance Networks based on ONVIF", Work shop on Multimedia Systems for Surveillance, (2011), pp. 420-423.

[9] C. H. Leiserson, "Fat-Trees: Universal Networks for Hardware-Efficient Supercomputing", IEEE Transactions on Computers, vol. C-34, no. 10, (1985), pp. 892-901.

[10] A. Paknikar, M. Kankanhalli and K. Ramakrishnan, "A Caching and Streaming Framework for Multimedia", ACM Multimedia, (2000), pp. 1-13.

[11] http://www.videolan.org/vlc.

[12] L. Adamic and B. Huberman, "Zipf's law and the Internet”, Glottometrics, vol. 3, (2002), pp. 143-150. 